\title{
Foliar Uptake of the Potencially Toxic Elements in Garlic Chive Leaves
}

\begin{abstract}
Eunseok Seo ${ }^{1}$, Seonghan Kim ${ }^{1}$, Jiwon Park ${ }^{2}$, Heejin $\mathrm{Lim}^{3}$, Nami Ha ${ }^{1}$, Jun $\mathrm{Lim}^{4}$, Jae Hong Lim ${ }^{4}$, Hyun Jin Park ${ }^{5}$ Ki Hean Kim ${ }^{1}$ and Sang Joon Lee ${ }^{1 *}$

${ }^{1}$ Department of Mechanical Engineering, Pohang University of Science and Technology (POSTECH), Pohang, South Korea, ${ }^{2}$ Department of Microbiology, Chungbuk National University, Cheongju, South Korea, ${ }^{3}$ Department of New Biology, Daegu Gyeongbuk Institute of Science and Technology (DGIST), Daegu, South Korea, ${ }^{4}$ Industrial Technology Convergence Center, Pohang Accerlerator Laboratory (PAL), Pohang, South Korea, ${ }^{5}$ National Institute for Nanomaterials Technology (NINT), Pohang University of Science and Technology (POSTECH), Pohang, South Korea
\end{abstract}

Contamination of vegetables due to the foliar uptake of atmospheric toxic elements could pose severe health risks. However, the uptake mechanisms of potencially toxic elements (PTEs) from the atmosphere and translocation by plant leaves remain unclear. In this study, carboxylic acid-functionalized water-soluble CdSe/ZnS quantum dot nanoparticles (QD NPs) were used as an experimental particle model of PTEs in the edible plant garlic chive (Allium tuberosum). A droplet of QD NP suspension was deposited to simulate the conditions of raindrops containing metal particles falling on a plant leaf. The 3D spatial distribution of QD NPs in plant leaves was measured using three complementary imaging techniques: synchrotron X-ray microcomputed tomography (micro-CT), nano-CT, and two-photon microscopy (TPM). The TPM and micro-CT results revealed that QD NPS deposited on garlic chive leaves penetrated the plant leaves. Nano-CT images showed that QD NPs are absorbed into mesophyll cells and phloem vessels. The results of TEM and TPM imaging demonstrated that QD NPs penetrate through the leaves and translocate in the direction of the stem. The use of these emerging imaging techniques improved the ability to detect and visualize NPs in a plant leaf. These observations also provide mechanistic insights into foliar metal uptake and their translocation and accumulation.

Keywords: potencially toxic elements, quantum dot, foliar uptake, CT, two-photon microscopy

\section{INTRODUCTION}

Modern industrialization has exacerbated air pollution by the potencially toxic elements (PTEs) worldwide (Biasioli et al., 2007; Pourret and Hursthouse, 2019).

The term "heavy metal" is a common term used for decades in the sciences (Pourret and Hursthouse, 2019). It is considered imprecise because there is no standardized definition for a heavy metal. All so-called "heavy metals" and their compounds may have relatively high toxicity [e.g., lead $(\mathrm{Pb})$ or cadmium $(\mathrm{Cd})]$ (Pourret and Hursthouse, 2019). Nevertheless, metals are not always toxic and some are not actually essential, and the balance between essential or toxic may be tilted depending on the dose and exposure level and the receiving organism/population [e.g., nickel or zinc (Zn)] (Pourret and Hursthouse, 2019). The toxicity of PTEs, such as Pb and Cd, depends on the differentiation and concentration of PTEs not only quantitatively but also qualitatively (Pourret and Hursthouse, 2019). The term "heavy metal" can cause a lot of misunderstandings and prejudices, so 
we define the fine metal particles as PTEs that can be mixed with pollutants in the air and potentially toxic.

PTEs can cause various complications and respiratory diseases in humans, in addition to posing severe secondary health risks via vegetable contamination (Shahid et al., 2017). Despite the serious issues caused by PTEs, the absorption of PTEs in edible plants has rarely been studied. PTEs emitted from industrial smelters are transported up to several kilometers away from their sources by wet or dry deposition (Douay et al., 2009; Shahid et al., 2013); they can accumulate in plant leaves through foliar transport after deposition of atmospheric particles on leaf surfaces (Schreck et al., 2012; Xiong et al., 2014). Unlike root transport of the PTEs, which has been widely studied (Pourrut et al., 2011), uptake of the PTEs from the atmosphere by plant leaves has not been fully explored (Tomasevic et al., 2005; Honour et al., 2009; Uzu et al., 2010). Furthermore, most studies on uptake of the PTEs have focused on incremental metal concentrations, without investigating the transport pathways involved (Hutchinson and Whitby, 1974; Lobersli and Steinnes, 1988; Ward, 1990; Salim et al., 1993; Little, 1995), or have been solely focused on biomonitoring fallout (Sloof, 1993; Conti and Cecchetti, 2001; Wolterbeek, 2002; Schreck et al., 2012). After deposition of atmospheric PTEs on plant leaves, the PTEs could penetrate plants via foliar transport (Shahid et al., 2017). However, the foliar pathways by which nanoparticles (NPs) enter plants are not well understood (Avellan et al., 2019). Stomatal and cuticular uptake pathways are two potential routes for the entry of NP into plant leaves (Avellan et al., 2019). Plants are known to take up and translocate metal NPs (Avellan et al., 2017). However, direct evidence of the physical processes of metal NP uptake and translocation in plants is scarce at the cellular level (Avellan et al., 2017; Shahid et al., 2017).

Studying NPs in plant matrices is a difficult task, and often requires the use of complementary tools to fully capture the NP fate at the cellular and organism level. Artifacts for the study can be generated through sample preparation, including cutting, labeling, and staining. X-ray computed tomography (CT) imaging techniques do not require cutting, labeling, or staining of the samples, thereby greatly reducing the risk of using artifacts in sample preparation. In particular, microcomputed tomography (micro-CT) and nano-CT enable visualization of foliar metal NP uptake by edible plants without additional contrast enhancement. In addition, two-photon microscopy (TPM) is a high-resolution non-destructive imaging technique that allows cross-sectional imaging-based 3D characterization of microscopic structures of test samples (Kampschulte et al., 2016).

In the present study, we investigated infiltration of the PTEs into plant leaves. We hypothesized that PTEs may be absorbed via cuticular and stomatal pathways. Carboxylic acidfunctionalized water-soluble $\mathrm{CdSe} / \mathrm{ZnS}$ quantum dot nanoparticles (QD NPs) were selected as an experimental model of PTEs. QD NPs have been used as contrast agents in various imaging techniques, such as confocal and TPM. The edible plant garlic chive (Allium tuberosum) was used as a model plant. A droplet of QD NPs suspension was deposited on a garlic chive leaf to simulate conditions of raindrops containing metal particles falling on the leaf surface. To characterize NP-plant interactions at the cellular level, the 3D spatial distribution of QD NPs in plant leaves was measured using synchrotron X-ray micro-CT, nano-CT, and TPM imaging techniques. These techniques provide mechanistic insights into QD NP-leaf interactions. Furthermore, the translocation behavior of the QD NPs absorbed through cuticular and stomatal pathways inside the plant leaves is discussed.

\section{MATERIALS AND METHODS}

\section{Materials}

Garlic chive (A. tuberosum) was purchased from a local market and grown in an experimental chamber. The experimental chamber was constantly maintained at $26 \pm 2^{\circ} \mathrm{C}$ and $50 \pm 5 \%$ humidity with a $12 \mathrm{~h}: 12 \mathrm{~h}$ light: dark photoperiod. LED lamps (PGL-E15, PARUS, Cheonan, Korea) were used to irradiate $430 \mathrm{~nm}: 460 \mathrm{~nm}: 660 \mathrm{~nm}$ light (Kim et al., 2016). The light intensity on the surface of the garlic chive leaves was approximately $450 \mu \mathrm{mol} / \mathrm{s} / \mathrm{m}^{2}$. We observed three leaves per experimental condition for each imaging technique. For observing the moving paths of QD NPs with TPM, one specimen was observed per condition owing to the prolonged time required to photograph the specimen.

CdSe/ZnS QD NP suspension (carboxylic acid-functionalized water-soluble $\mathrm{CdSe} / \mathrm{ZnS}$ core-shell type QD NPs, fluorescence $\lambda_{\text {em }}$ of $560 \mathrm{~nm}, 1 \mathrm{mg} / \mathrm{ml}$ in $\mathrm{H}_{2} \mathrm{O}$, and approximately $5 \mathrm{~nm}$ in diameter) was purchased from Sigma-Aldrich (United States). All materials used in the sample preparation were purchased from Sigma-Aldrich.

\section{Measurement of Zeta Potential and Hydrodynamic Diameter}

The hydrodynamic particle size $(\square)$ and zeta potential $(\zeta)$ of the QD NPs were estimated using Zetasizer Nano ZS analyzer (Malvern Instruments, Worcestershire, United Kingdom). When measuring the $\zeta$ using a zetasizer $S$, the refractive index (RI) and absorbance $(A)$ of the QD NPs at a wavelength of $633 \mathrm{~nm}$ were estimated as 0.13 and 0 , respectively. When measuring the $\square$ using a zetasizer $\mathrm{Z}$, the RI and A were set as the material properties of the core part of QD NPs (CdSe), and the values were 0.25 and 0 , respectively. Approximately $10 \mu \mathrm{L}$ QD suspension was diluted in a cuvette using $4 \mathrm{ml}$ deionized water to satisfy the scale of nanomoles. The measurements were conducted 20 times to measure the $\zeta$, and 10 times to determine the $\square$ at $25^{\circ} \mathrm{C}$. The results are presented as the mean, and error bars indicate the standard deviation (SD). The $\square$ and $\zeta$ values of QD NPs in suspension are shown in Supplementary Figure S2.

\section{Scanning Electron Microscopy Imaging}

Detailed morphological features of the leaf surface and stomata of garlic chive were obtained using a field emission scanning electron microscope (JEOL JSM-7401F, JEOL, Tokyo, Japan) 


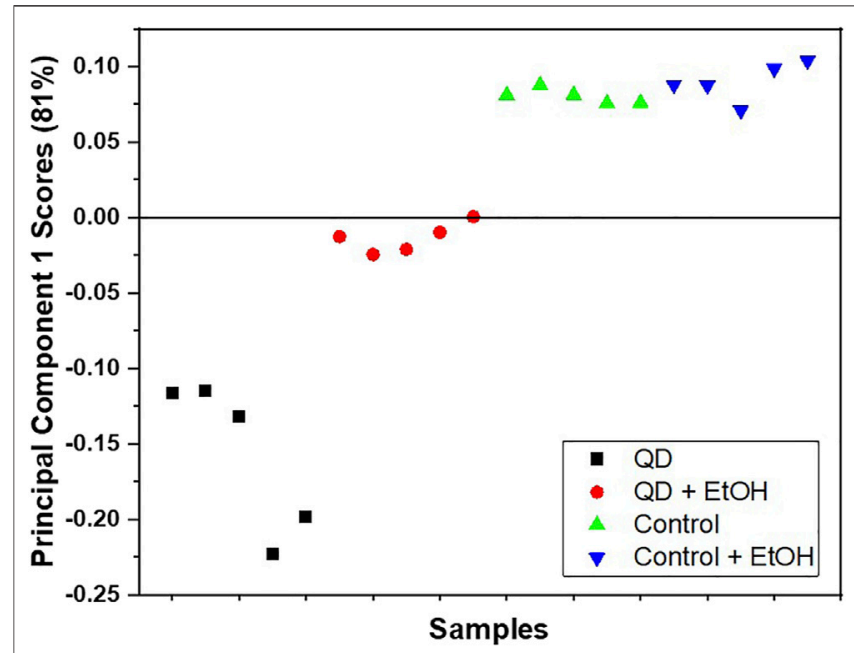

FIGURE 1 | Score plot of PC1 obtained via PCA of the positive ion TOFSIMS spectra for the garlic chive leaf surfaces treated with QD NPS ( $\bullet)$, ethanol and QD NPs (-), the control $(\mathbf{\Delta})$, and the ethanol-treated control $(\boldsymbol{\nabla})$. Five data points were obtained for each surface.

after coating them with platinum (SC7640 model, Quorum Technology, United Kingdom) for $30 \mathrm{~s}$ to avoid any charging effect. SEM images of a typical garlic chive leaf are shown in Supplementary Figures S3B,C.

\section{Optical Imaging}

A strip of garlic chive leaf was fixed with 3.5\% formaldehyde solution at room temperature for $24 \mathrm{~h}$. After fixation, the sample was embedded in paraffin in accordance with the standard protocol and cut into slices of $8 \mu \mathrm{m}$ thickness using a rotary microtome (Finess ME, Thermo). The sliced samples were deparaffined and stained with $1 \% \mathrm{w} / \mathrm{v}$ Alcian blue $(\mathrm{pH} 2.5)$ and $0.1 \% \mathrm{w} / \mathrm{v}$ Safranin $\mathrm{O}$ solution. The morphological features of the stained samples were observed using an optical microscope (Zeiss Axiovert 200, Germany) with a $10 \times$ objective lens. An optical image of a cross-sectional garlic chive leaf is shown in Supplementary Figure S3D.

\section{Time-of-Flight Secondary Ion Mass Spectrometry Analysis}

To analyze the surface of the sample without pretreatment, the garlic chive plant leaves were investigated after exposure of the leaves to a $2 \mu \mathrm{L}$ droplet $(1 \mathrm{mg} / \mathrm{ml})$ of QD NPs for 1 day. Garlic chive not exposed to QD NPs was used as the control. A carbon tape was attached onto silicon wafer chips cut $1 \mathrm{~cm}$ by $1 \mathrm{~cm}$, and the prepared samples were cut and attached.

In order to analyze the effect of the sample pretreatment for micro-CT imaging, the control and QD NPs treated garlic chive leaves were fixed using $3.5 \%$ formaldehyde solution at room temperature for $24 \mathrm{~h}$. The fixed leaves were then dehydrated by immersing them in an ethanol series of 25, 50, 75, and $100 \%$ for $30 \mathrm{~min}$ each. A carbon tape was attached onto silicon wafer chips cut $1 \mathrm{~cm}$ by $1 \mathrm{~cm}$, and the prepared samples were cut and attached.

ToF-SIMS analysis was conducted on a ToF-SIMS 5-100 instrument (ION-TOF, Münster, Germany) using a pulsed $30 \mathrm{keV} \mathrm{Bi}_{3}{ }^{+}$primary ion beam in the spectrometry mode, with a primary ion dose of $1.64 \times 10^{12} \mathrm{ions} / \mathrm{cm}^{2}$ across a $100 \times 100 \mu \mathrm{m}^{2}$ area for positive ions. An electron flood gun supplied low-energy electrons onto the surface of the samples for charging compensation during analysis. Internal mass calibration for all the ToF-SIMS spectra was performed using the peaks of $\mathrm{CH}_{3}{ }^{+}$, $\mathrm{C}_{2} \mathrm{H}_{3}{ }^{+}, \mathrm{C}_{3} \mathrm{H}_{5}{ }^{+}$, and $\mathrm{C}_{4} \mathrm{H}_{7}{ }^{+}$before further analysis.

Multivariate analysis was conducted through principal component analysis (PCA) using MATLAB (Version R2021a, MathWorks Inc., Natick, MA, United States) and NBToolbox (Version 2.9, Daniel Graham Ph.D., NESAC/BIO, University of Washington). A list of peaks was detected by employing an automatic peak search at $\mathrm{m} / \mathrm{z}<500$. The peak areas were normalized by the total ion counts. The data were treated with mean centering as a preprocessing step before PCA.

\section{Micro-CT Imaging}

The interactions of CdSe/ZnS QD NPs and garlic chive plant leaves were investigated after exposure of the leaves to a $2 \mu \mathrm{L}$ droplet $(1 \mathrm{mg} / \mathrm{ml})$ of QD NPs for $1,3,5,7$, and 21 days (Supplementary Figure S4). A $1 \mu \mathrm{L}$ droplet was difficult to precisely drop using a pipette, while $3 \mu \mathrm{L}$ was too large and overflowed to the outside of the leaf. Therefore, we selected a droplet size of $2 \mu \mathrm{L}$ for the analysis. Garlic chive not exposed to QD NPs was used as the control. The plant leaves were fixed using $3.5 \%$ formaldehyde solution at room temperature for $24 \mathrm{~h}$. The fixed leaves were then dehydrated by immersing them in an ethanol series of $25,50,75$, and $100 \%$ for 30 min each. The sample and $100 \%$ ethanol were inserted into a heat-sealed pipette tip. The open part of the tip was blocked with a lid made of PDMS (Dow Corning, United States) polymer to prevent the ethanol from evaporating.

The 3D morphological structures of garlic chive leaves soaked in ethanol were observed by synchrotron X-ray micro-CT at the 6C biomedical imaging beamline of Pohang Accelerator Laboratory (PAL, Pohang, Korea). A monochromatic X-ray beam of $14 \mathrm{keV}$ was passed through the sample, and X-ray images were captured by a high-speed camera (PCO AG, Germany) located at $40 \mathrm{~mm}$ behind the sample. The test sample was fixed on a rotary stage (ABRS-150 MP-M-AS, Aerotech, Inc., Pittsburgh, PA, United States) to collect tomographic slice images. The microscope (Optique Peter, Lentilly, France) used for X-ray imaging was composed of a $5 \mu$ m-thick terbium-doped $\mathrm{Lu}_{2} \mathrm{SiO}_{5}$ scintillator (FEE), objective lenses (10×: UPLSAPO10X2, 20×: UPLSAPO20X2, and 40×: UPLSAPO40X2, Olympus, Japan), and a CMOS camera (Andor Zyla). A total of 901 projection images were consecutively captured by rotating the rotary stage from 0 to $180^{\circ}$ at intervals of $0.2^{\circ}$ to acquire the corresponding $3 \mathrm{D} \mathrm{CT}$ image. The $3 \mathrm{D}$ volume images reconstructed from the captured tomographic projection images were rendered using Octopus software (inCT, Gent, Belgium) and Amira image analysis software (FEI, Hillsboro, OR, United States) (Lee et al., 2019; 

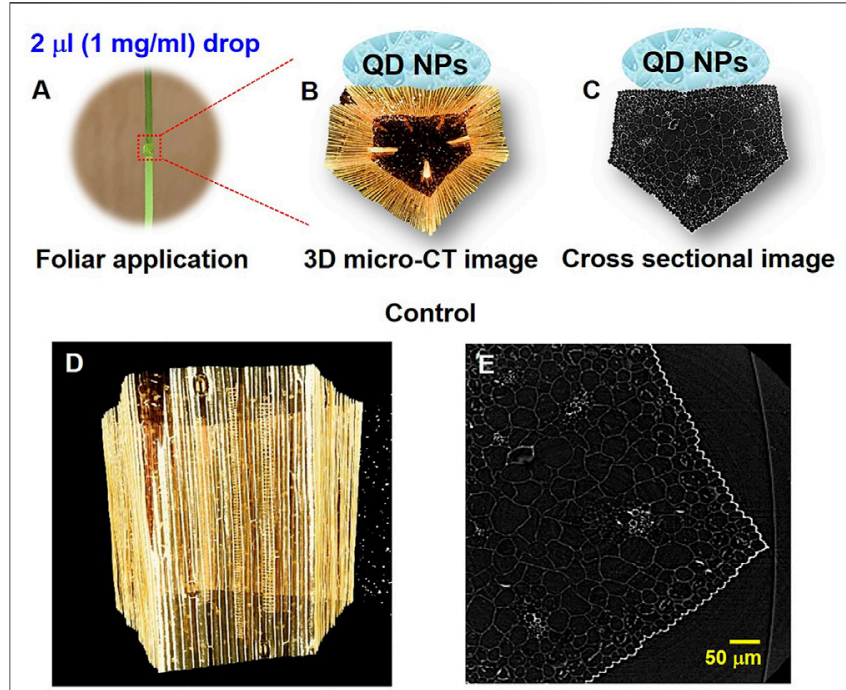

QD treatment for $3 \mathrm{~d}$
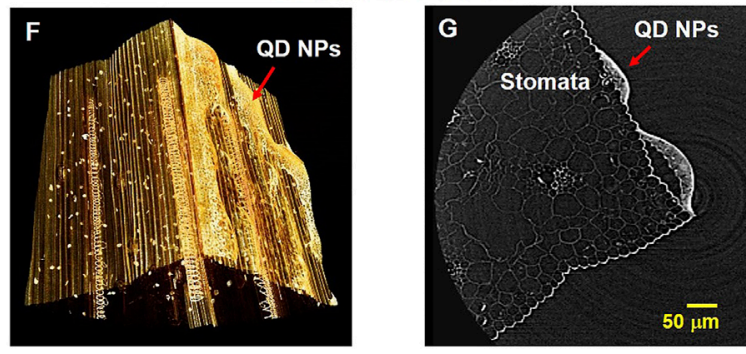

FIGURE 2 |Adsorption of QD NPs on the abaxial surface of a garlic chive leaf. (A) QD NP suspension (2 $\mu \mathrm{L}$ droplet) was deposited on the abaxial side of the garlic chive leaf. 3D (B) and cross-sectional $(\mathbf{C})$ images $(10 \times)$ of the fixed garlic chive leaf based on micro-CT. (D,E) 3D and cross-sectional microCT image (20x) of the control garlic chive leaf. (F,G) QD NP deposition and penetration through stomata. QD NPs translocated across the leaf epidermal barrier through the stomata. Red arrowheads indicate QD NPs.

Seo et al., 2020). The micro-CT imaging technique employed in this study has a spatial resolution range of $0.165-0.65 \mu \mathrm{m}$.

\section{Nano-CT Imaging}

The interactions of CdSe/ZnS QD NPs and garlic chive plant leaves were investigated after exposure of the leaves to a $2 \mu \mathrm{L}$ droplet $(1 \mathrm{mg} / \mathrm{ml})$ of QD NPs for 1, 3, 5, 7, 14, and 21 days. It is relatively simple to use micro-CT to photograph specimens that are larger than the field of view (FOV); however, it is very difficult to centralize nano-CT for tomo imaging when photographing specimens larger than the FOV. Therefore, at the maximum, the leaves used for nano-CT should be cut to the size of the FOV. The cut leaves were fixed using 3.5\% formaldehyde solution at room temperature for $24 \mathrm{~h}$. The fixed leaves were dehydrated in a graded ethanol series up to $100 \%$ and then embedded in Spurr's resin for X-ray Zernike phase contrast nano-CT imaging. The X-ray used in micro-CT is a white beam, and the beam strength is lower than that of the mono beam used in nano-CT. Therefore, it is possible to observe the specimen in ethanol without damaging it. If nano-CT images of this specimen are taken, the specimen will be burned or damaged by the strong
X-ray mono beam. Thus, the specimen used for nano-CT was pretreated with a resin to prevent damage.

Nano-CT measurements were conducted at the 7C X-ray nano-imaging beamline of PAL. This beamline was specially designed for spectral microscopy and nano-CT by utilizing zone plate-based full-field transmission X-ray microscopy. The monochromatic X-ray beam at $9.3 \mathrm{keV}$ was selected using a liquid nitrogen-cooled double-crystal monochromator and focused using a rhodium-coated horizontal focusing plane mirror and a vertical focusing plane mirror. A golden objective zone plate of $30 \mathrm{~nm}$ outermost zone width, $300 \mu \mathrm{m}$ diameter, and $0.9 \mu \mathrm{m}$ thickness was used for nano-CT. The Zernike phase contrast method was employed by adopting a holed golden film phase plate with a thickness of $960 \mathrm{~nm}$ to obtain high-contrast X-ray images. A scintillator-coupled optical microscope incorporating a thin scintillation crystal $(20 \mu \mathrm{m}$ thick GAGG:Ce), a $20 \times$ optical objective, and a high-end CCD with $2,048 \times 2,048$ pixels was used as a detector. In this experiment, the FOV was $50 \mu \mathrm{m}$ and the effective pixel size (with two bins) was equivalent to $50 \mathrm{~nm}$. For tomographic scanning, 721 projections were acquired with $1 \mathrm{~s}$ of exposure time. Since the FOV is considerably smaller than $50 \mathrm{~nm}$, the vibrations caused by stage rotation can be transmitted to the specimen. In this case, additional calibration is required after each experiment. However, for the present experimental conditions $(\mathrm{FOV}=50 \mu \mathrm{m})$, there was no such problem, and no further correction was required. Tomographic reconstruction was conducted by adopting the filtered back projection algorithm method (Octopus software). Segmentation and visualization of the $3 \mathrm{D}$ data were carried out using the commercial software package Avizo (Thermo Fisher Scientific, United States).

\section{TPM Imaging}

The interactions of CdSe/ZnS QD NPs and garlic chive leaves were investigated after exposure to a $2 \mu \mathrm{L}$ droplet $(1 \mathrm{mg} / \mathrm{ml})$ of QD NPs for 1, 3, 5, 7, and 21 days (Supplementary Figures S4, S5). A custom-built two-photon microscope equipped with a TiSapphire laser (Chameleon Ultra II, Coherent) light source was used in this study for live imaging of QD NPs-leaf interactions. The excitation wavelength was set at $900 \mathrm{~nm}$. TPM used a $20 \times$ objective lens (Olympus UPlanSApo) with a numerical aperture of 0.75 . Emission fluorescence signals were spectrally resolved at two channels: $580-640 \mathrm{~nm}$ for the chlorophyll signal and 505-545 $\mathrm{nm}$ for the QD signal. These signals were detected by two photomultiplier tubes (H7421-40, Hamamatsu, Japan) in the photon counting mode. The FOV was $300 \times 300 \mu \mathrm{m}$ in the $\mathrm{xy}$ plane with $512 \times 512$ pixels. The imaging speed was 0.1 frames per second. Volume scanning was conducted by stepwise increment in the $\mathrm{z}$-direction at intervals of $2 \mu \mathrm{m}$. The experimental setup for TPM imaging is shown in Supplementary Figure S6. Amira image analysis software (FEI) was used to reconstruct 3D images of QD NPs in plant leaves.

\section{TEM Imaging}

TEM imaging for QD NP characterization was conducted using the transmission electron microscope (JEOL JEM-2200FS) of the 

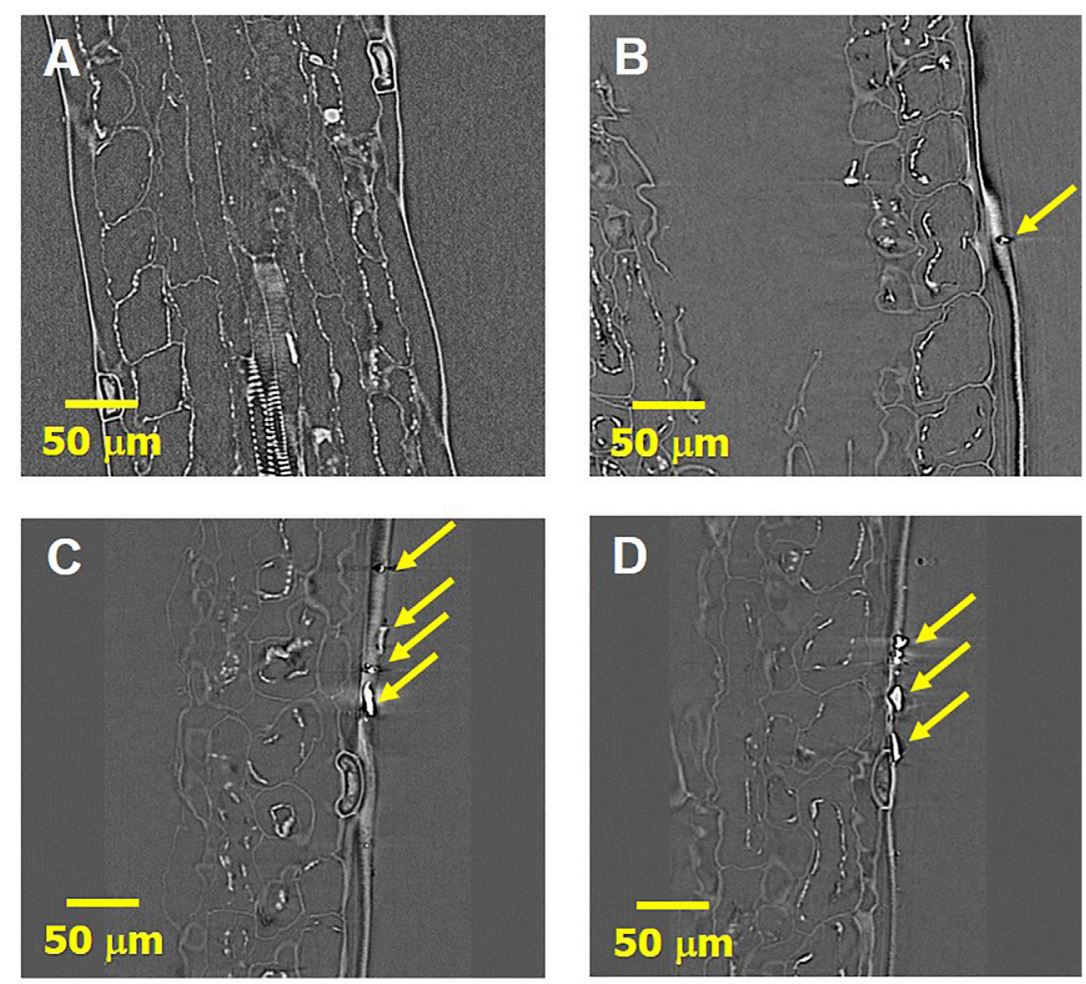

FIGURE 3 | Penetration of QD NPs via cuticlar cracks (physical barrier of foliar QD NP uptake). X-ray micro-CT images of the control (A) and QD NP-treated (B-D) garlic chive leaves for 21 days. 2D slices virtually extracted from the reconstructed 3D images. (A-D) Longitudinal section images (40x). (B-D) QD NPs translocate across the leaf epidermal barrier. Yellow arrowheads indicate QD NPS.

National Institute for Nanomaterials and Technology, Pohang. The TEM images of QD NPs are shown in Supplementary Figure S1. TEM imaging was performed for the control and QD-treated leaves. The leaves were prefixed in $3.5 \%$ formaldehyde solution and post-fixed in 1\% osmium tetroxide buffer for $24 \mathrm{~h}$ at $4^{\circ} \mathrm{C}$. Then, the samples were dried using a graded series of ethanol and then further dehydrated in propylene oxide and Spurr's resin. Subsequently, ultrathin sections $(90-120 \mathrm{~nm})$ of the leaves were collected on formvar-coated grids and counterstained with $2 \%$ uranyl acetate and Reynolds' lead citrate (MT-X, RMC, Tucson, AZ, United States). Finally, the samples were observed via TEM (JEOL JEM-1011, JEOL) at $80 \mathrm{kV}$.

\section{RESULTS}

\section{ToF-SIMS Analysis}

The coating of QD NPs can greatly influence their behavior. In this study, QD NPs coated with carboxyl groups were used due to their ability to easily stick to and move in plant leaves, as carboxyl groups contain water. As expected, QD NPs were observed to adhere well to the leaf surface. In addition, QD NPs that were adhered to the leaf surface via the pretreatment process exhibited a slight loss during the washing process.
ToF-SIMS showed that most of the QD NPs were firmly adhered to the leaf surface. Figure 1 shows a plot of principal component 1 (PC1) scores obtained from the PCA of positive ion TOF-SIMS spectra. PC1 captured $81.2 \%$ of variances in the dataset. PC1 scores show the main distinction between the QD and control groups. In particular, QD groups are clearly distinct from other groups in the score plot; however, the control and ethanol-treated control groups were not easily distinguishable from each other. The PC1 scores of the ethanol-treated QD group are located in the middle between the quantum dot and control groups. These results imply that most QD NPs were not removed by ethanol treatment, and that there was no damage to the leaf surface in the control group.

\section{Foliar QD NP Uptake: Micro-CT Imaging}

The absorption of QD NPs on the surface of garlic chive leaves was experimentally investigated (Figure 2). As shown in Figures 2A-C droplet $(2 \mu \mathrm{L})$ of QD NPs was deposited on the abaxial side of a garlic chive leaf. The reconstructed $3 \mathrm{D}$ image (Figure 2B) showed a typical morphological structure of the garlic chive leaf. The cross-sectional image (Figure 2C) showed the internal morphological structure of the leaf. A possible mechanism of QD NP absorption through stomatal openings is depicted in the cross-sectional micro-CT images of 

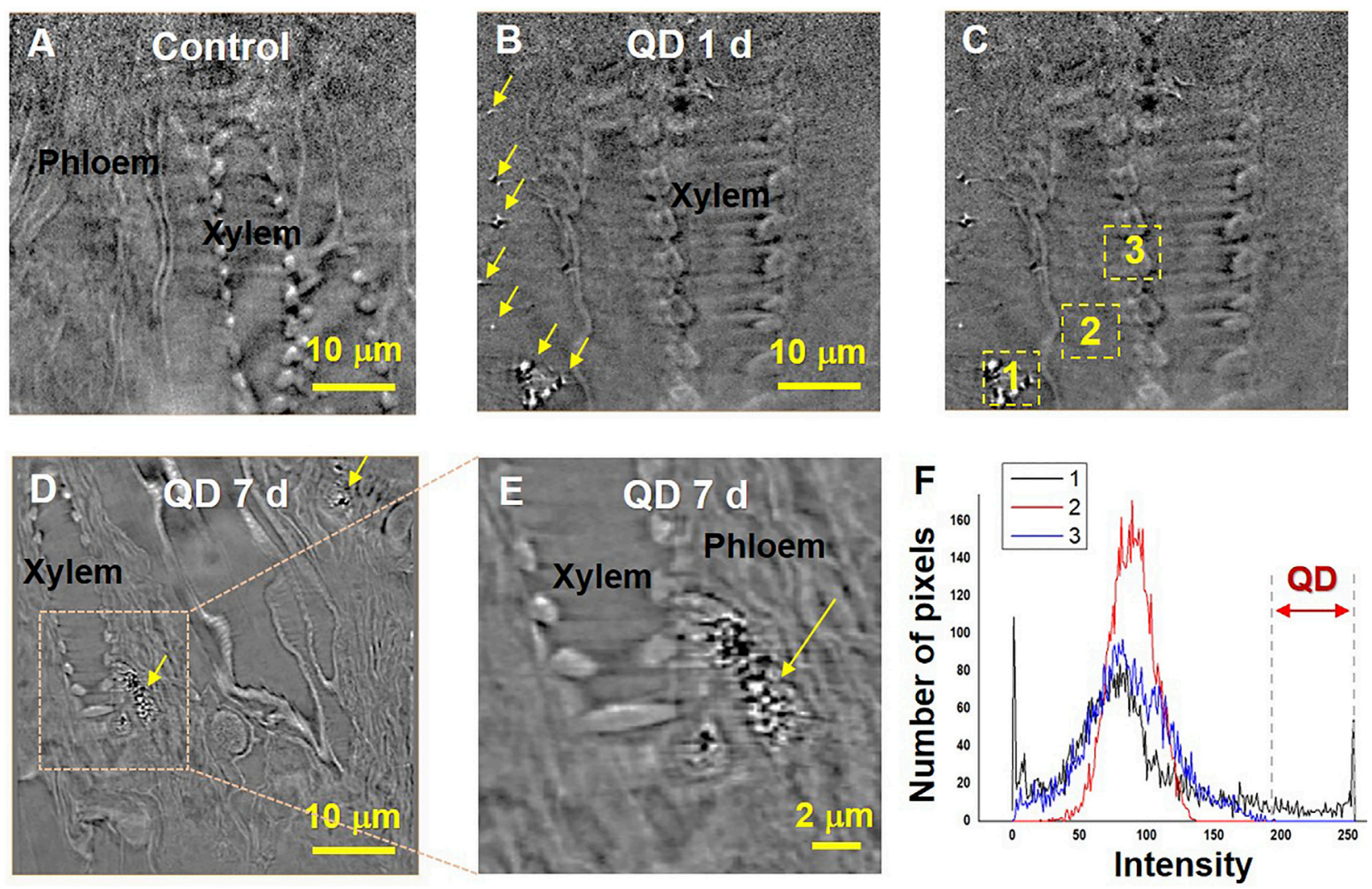

FIGURE 4 | Translocation of QD NPs within garlic chive plants after their foliar uptake. X-ray images of the control (A) and QD-exposed leaves (B-E) captured by nano-CT. 2D slices extracted from the reconstructed 3D images. Yellow arrowheads indicate QD NPS. QD $1 \mathrm{~d}$ and 7 days represent X-ray images captured at 1 and 7 days after foliar exposure to $1 \mathrm{mg} / \mathrm{ml}$ QD suspension ( $2 \mu \mathrm{L}$ drop deposition), respectively. To determine the existence of QD, area histograms (F) of the three different regions (C) were compared. The two way red arrow (the region of intensity 200-250) indicates a range of brightness regions only for QD NPS.

Figure 2G. QD NPs were accumulated on the leaf surface where the QD NP suspension was deposited. They were then translocated across the epidermal barrier of the leaf through the stomata. As illustrated in Figures 3B-D, the QD NPs were entrapped in the epidermal cell walls. These images show that QD NPs were translocated across the leaf epidermal barrier through cuticular pathways.

\section{QD NP Translocation in Garlic Chive Leaves: Nano-CT Imaging}

Three garlic chive models, the control, and garlic chive leaves treated with QD NPs were prepared according to the fixation protocols for contrast-enhanced X-ray imaging. Specimens must be cut to a size smaller than the FOV to effectively capture nanoCT images; therefore, it was difficult to obtain the QD NP images within the cut area. QD NPs were observed in the specimens treated with QDs for 1, 3, 5, and 7 days. We used the images of specimens treated with QDs for 1 or 7 days as the representative images in Figure 4.

The translocation of QD NPs in the garlic chive leaves was examined by employing nano-CT imaging (Figure 4). As shown in Figure 3B, QD NPs were observed in the interfacial regions of the mesophyll cells of the model treated with QD NPs for 1 day, but not in the control model (Figure 4A). After foliar uptake, the QD NPs were diffused into mesophyll cells and translocated through the apoplastic or symplastic pathway.

Next, we determined whether QD NPs could translocate from the leaf to the root through phloem vessels. The possibility of QD NP translocation was identified by observing several longitudinal section images of the garlic chive leaves treated with QD NPs for 7 days. As shown in Figures 4D,E, QD NPs were accumulated in phloem vessels; this was verified by the areas of interest and the area histograms shown in Figures 4C,F.

\section{Foliar QD NP Uptake and Translocation: TPM Imaging}

The 3D spatial distributions of the absorbed QD NPs were examined using TPM imaging. As TPM permits direct observation of living plant cells, the internalization of QD NPs by plant cells was detected. However, TPM has poor lateral resolution. The reconstructed $3 \mathrm{D}$ image in Figure $\mathbf{5 A}$ shows the morphological structure of chlorophyll in the leaf epidermis. 

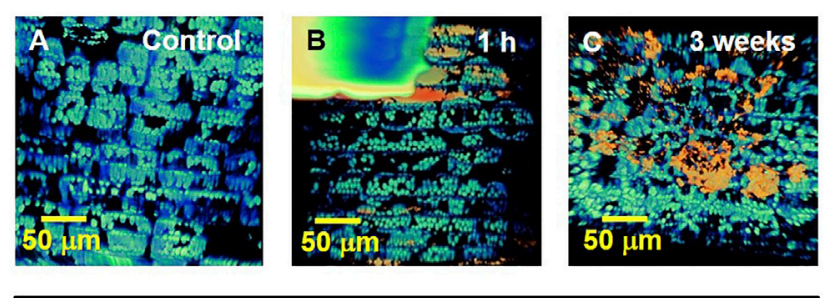

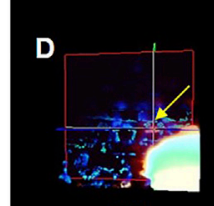

3D

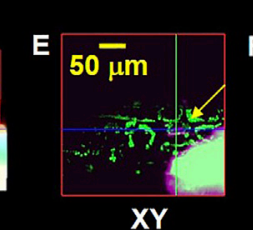

$\mathbf{X Y}$
QD treatment for $\mathbf{5} \mathbf{d}$

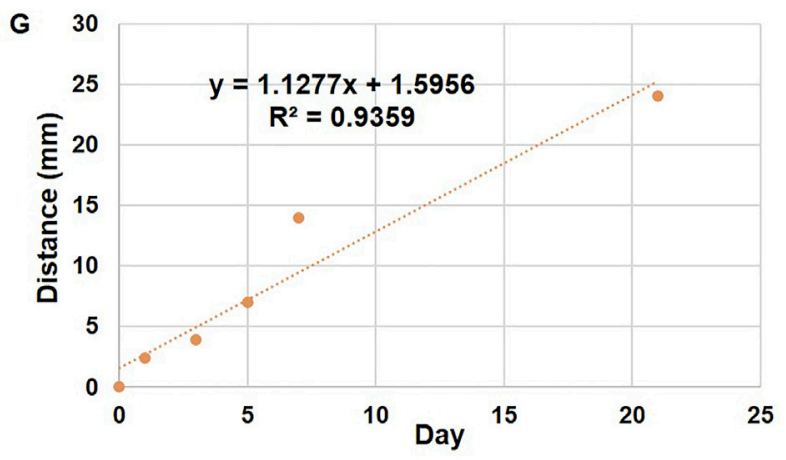

FIGURE 5 | Interaction of QD NPS with garlic chive leaves after drop deposition. QD NP-leaf interactions were investigated after 0, 1, 3, 5, 7, and 21 days of exposure of the leaves to a $2 \mu \mathrm{L}$ droplet $(1 \mathrm{mg} / \mathrm{ml}$ ) of QD NPs. (A) $3 \mathrm{D}$ reconstruction image showing chlorophyll (blue fluorescence) distribution in the control garlic chive leaf. A droplet of QD NP suspension was deposited on the abaxial side of the leaf. After being exposed from $1 \mathrm{~h}(\mathbf{B})$ to 3 weeks (C), QD NP absorption (red fluorescence) into the garlic chive leaf was observed. (D) 3D image showing QD NP uptake into the garlic chive leaf through the stomatal pathway. Cross-sectional images in the xy (E) and yz (F) planes showing the internal morphological structures of garlic chive leaf. Yellow arrowheads indicate stomata. (G) TPM data analysis of QD NP uptake in plant leaves. Y-axis represents the translocation distance of QDs in the leaves over time. The $X$ axis represents the elapsed time after the QD NP droplet treatment.

Figure 5B illustrates a typical TPM image of the drop deposition area on a garlic chive leaf after $1 \mathrm{~h}$ of exposure. At the location of QD NP deposition, the light intensity was saturated owing to densely distributed QD NPs in the region. As the droplet evaporated, QD NPs were translocated to the regions near the leaf surface (Figure 5C).

The cross-sectional images (Figures 5D-F) show the internal morphological structure of the leaf treated with QD NPs for 5 days. These images show the possibility of QD NP absorption by stomatal openings. The QD NPs were translocated across the leaf epidermal barrier through the stomata. The presence of QD NPs in the region of stomatal guard cells or pores indicated that they were translocated through the stomatal pathway.

In this experiment, we did not observe any fluorescence in the cuticle layer. Therefore, it is not possible to determine whether cuticular uptake occurred. Instead, QD NPs appeared inside the leaves (Supplementary Figure S7C). We placed a droplet of QD NP suspension on the leaf surface and took a cross-sectional image along the $\mathrm{z}$-axis to observe whether QD NPs infiltrate the leaf surface and translocate inside it. As illustrated in the $\mathrm{XZ}$ and $\mathrm{YZ}$ cross-sectional images in Supplementary Figure S7C, QD NPs were located between chlorophylls. These results support the idea that QD NPs exist inside the plant leaf. The XY cross-sectional image in Supplementary Figure S7C shows the area where QD NPs appear in the XY and YZ cross-sections. For further analysis, we sought to confirm whether QD NPs exist inside the leaf by observing the $\mathrm{XZ}$ and $\mathrm{YZ}$ section images (Supplementary Figure S7D). Since the observed QD NPs are > one pixel in the image, we infer that they may comprise aggregations of several QD NPs.

TPM analysis was conducted to analyze the distance that the absorbed QD NPs translocated along the longitudinal axis of the leaves (Figure 5G). We prepared all specimens simultaneously and conducted TPM imaging at 1, 3, 5, 7,21 days after dropping QD NP droplets on plant leaf samples. The control specimen was first observed, and on the same day, five samples were prepared using a drop of QD NP suspension on the leaf surface. After preparing six samples simultaneously, the control specimen was first observed with TPM. After 1 day, we observed a test sample exposed to one drop of QD NP suspension with TPM. All specimens were prepared using this procedure for a total of 21 days. We captured images along the $\mathrm{z}$-axis while moving the stage from the area where the QD NP suspension was dropped on the leaf toward the stem. Supplementary Figure S7C,D show the area where QD NPs were agglomerated. The size of the observed QD NP cluster was very small; most of the observed QD NP clusters had similar sizes, as shown in Supplementary Figure S7C,D. The QD NPs slowly moved from the leaves towards the stem over time (Figure 5G).

\section{Foliar QD NP Uptake and Translocation: TEM Imaging}

TEM was used to detect spatial distributions of QD NPs inside garlic chive leaves at the subcellular level. The specimen must be cut to a thickness of 90-120 nm for TEM imaging, which made it difficult to locate specimens with QD NPs. Figures 5B,C show the internalized QD NPs in the intracellular spaces of the cytoplasm and epidermal cells. The black dots in Figure 6D represent the presence of QD NPs. The results indicate that QD NPs were translocated across the epidermal barrier of the plant leaves.

\section{DISCUSSION}

The foliar uptake and translocation pathways of QD NPs after foliar exposure are summarized in the schematics depicted in Figure 7. The leaves of higher plants are protected by waxy cuticles, which protect against water loss and uncontrolled exchange of other solutes; thus, the cuticle is considered the first natural barrier preventing NPs from entering substrate 

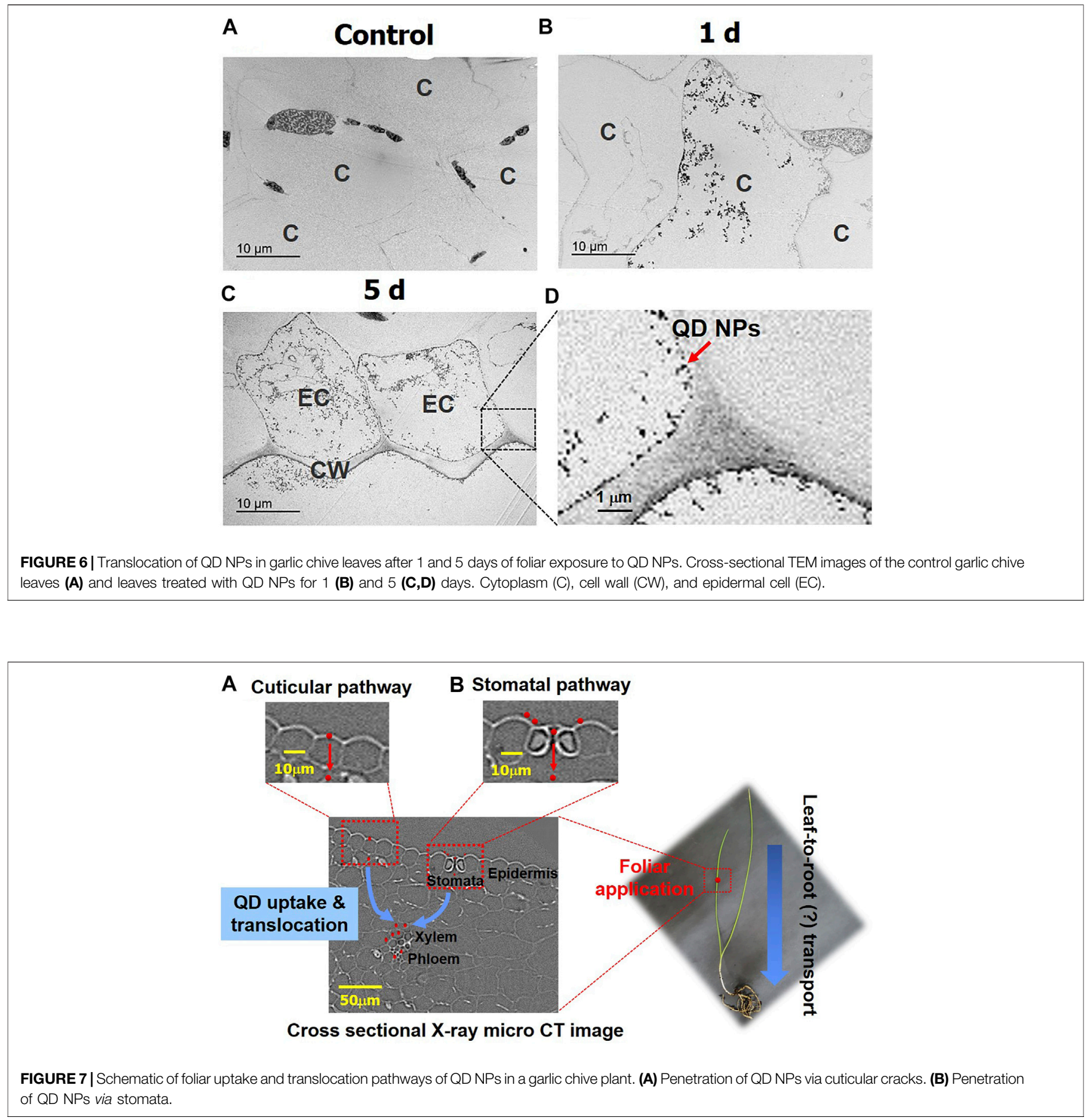

tissues (Pollard et al., 2008). The two routes for solute uptake across cuticles (cuticular pathway) are diffusion and permeation (lipophilic pathway) for nonpolar solutes and polar aqueous pores (hydrophilic pathway) for polar solutes, with an estimated effective size of $0.6-4.8 \mathrm{~nm}$ (Popp et al., 2005; Eichert and Goldbach, 2008; Eichert et al., 2008). Accordingly, NPs that are smaller than $4.8 \mathrm{~nm}$ in size may directly penetrate through the cuticles via the cuticular pathway, while many studies report foliar uptake and accumulation of NPs $>5 \mathrm{~nm}$ ( $\mathrm{Lv}$ et al., 2019). NP uptake can occur through the pores of leaf surfaces, including stomata (Avellan et al., 2021), and stomatal NP uptake is likely to be dependent on the plant morphology and physiological status (Avellan et al., 2021). Despite this information, the uptake pathway for these NPs remains unclear ( $\mathrm{Lv}$ et al., 2019). The cuticles and stomata are the 
two main pathways for the entry of nanomaterials through the leaf epidermis into mesophyll cells ( $\mathrm{Hu}$ et al., 2020). Inside leaves, nanomaterials can translocate via the extracellular (apoplastic) and/or intracellular (symplastic) pathways of mesophyll cells (Hu et al., 2020).

Although foliar NP uptake has been observed in many studies, none have provided direct evidence to support the subsequent pathway by which NPs are translocated through the phloem vessels of plants ( $\mathrm{Lv}$ et al., 2019). Wang et al. reported that small NPs (four metal oxide NPs of 24-47 nm in size) could penetrate watermelon leaves through the stomatal pathway, and metal elements were detected in the shoots and roots (Wang et al., 2013). They concluded that NPs passed through the shoots and finally reached the roots through phloem sieve tubes. Given that plant vascular systems are noncirculatory, nanomaterials moving downward in phloem vessels do not circulate back to their original sites through xylem vessels (Lough and Lucas, 2006). In the present study, QD NPs were accumulated in phloem vessels. This finding indicated that PTEs were transported to other parts of the plant via phloem vessels in the same manner as photosynthates after foliar penetration (Shahid et al., 2017). Therefore, phloem vessels are used as a means for NP translocation (Lv et al., 2019).

Foliar uptake of the PTEs is highly dependent on various factors, such as the physicochemical characteristics of cuticles and PTEs, the morphology and surface area of the test plant leaves, the chemical and physical forms of the adsorbed PTEs, the surface texture (pubescence and roughness) and habitus (deciduous or evergreen) of plant leaves, exposure duration, environmental conditions, and gas exchange (Beckett et al., 2000a; Beckett et al., 2000b; Shahid et al., 2017). Previous studies have reported that the ability of plants to absorb metal NPs depends on their different epidermal properties (Tomasevic et al., 2005; Luo et al., 2011).

In this study, to easily track the moving pathways of QD NPs, garlic chive was selected as a model plant as it possesses a simple, thin, and long leaf structure. Since the foliar characteristics (e.g., cuticle length and composition; compost leaves; presence of trichomes) have a strong influence on the quality of the experimental data, the testing of these methods in various plant species or ecological types will further help us to understand the interactions of fine particles and the mechanism of their behaviors within the plant.

When washing ethanol, QD NPs attached to the surface may fall off slightly. However, as can be seen from the micro-CT images, a very large amount of QD NPs stick to the leaf surface without being removed by washing. Since sample preparation bias can occur and cannot be avoided, dobule-check was performed using various detection, semi quantification and imaging methods like TOF-SIMS, PCA analysis, TPM and TEM imaging.

In future studies, we aim to investigate the mechanisms by which PTEs contaminate the leaves of various plants with diverse shapes and structures. These studies will facilitate better understanding of the uptake mechanisms of PTEs on plant leaves. It is important to show the effect of foliar application of metals on plant growth, pigment contents, and antioxidant enzymes to confirm that their uptake can cause adverse effects on the environment and human health.

\section{CONCLUSION}

Despite several recent studies on foliar absorption of the PTEs, the mechanism underlying foliar uptake of the PTEs in plant leaves is not well understood. In the present study, micro-CT, nano-CT, and TPM were employed to explore foliar uptake of the PTEs. Carboxylic acid-functionalized water-soluble CdSe/ZnS QD NPs were used as an experimental model of PTEs. The absorption of QD NPs into garlic chive leaves was experimentally investigated using the droplet deposition method, which simulates conditions of raindrops containing PTEs falling on the abaxial leaf surface.

The results of TPM and micro-CT revealed that QD NPs deposited on the garlic chive leaves entered the plant by penetrating through the stomatal and cuticular pathways. NanoCT images demonstrated that QD NPs were absorbed into mesophyll cells and phloem vessels. The results of TEM and TPM imaging experiments demonstrated that QD NPs were translocated from the leaf surface to the stem. The use of complementary 3D imaging techniques was helpful for elucidating the interactions between metal NPs and plant leaves. Taken together, the findings of this study aid in elucidating translocation and accumulation of the PTEs in plants, which is important to assess the harmful impacts of the PTEs on the environment and human health.

\section{DATA AVAILABILITY STATEMENT}

The original contributions presented in the study are included in the article/Supplementary Material, further inquiries can be directed to the corresponding author.

\section{AUTHOR CONTRIBUTIONS}

ES, SK, JP, HL, NH, and HP performed the experiments. JL and JHL provided guidance and technical expertise on $\mathrm{X}$-ray imaging. ES, SK, JP, HL, and NH analyzed the data. ES, SK, JP, HL, NH, and JL wrote the manuscript. SL supervised the project and finalized the manuscript. All authors contributed to reading and editing the manuscript.

\section{FUNDING}

This study was supported by the National Research Foundation of Korea through a grant funded by the Korean Government (No. SRFC-MA1401-07).

\section{ACKNOWLEDGMENTS}

We thank Jaewoo Ahn for valuable assistance with the TEM imaging experiments, which were performed at the TEM 
laboratory of the POSTECH Biotech Center. The authors are also grateful to the staff and supporting groups of the $6 \mathrm{C}$ X-ray medical imaging beamline and 7C nano-imaging beamline of the Pohang Acceleration Laboratory (Pohang, Korea) for their assistance during the X-ray experiments.

\section{REFERENCES}

Avellan, A., Schwab, F., Masion, A., Chaurand, P., Borschneck, D., Vidal, V., et al. (2017). Nanoparticle Uptake in Plants: Gold Nanomaterial Localized in Roots of Arabidopsis thaliana by X-ray Computed Nanotomography and Hyperspectral Imaging. Environ. Sci. Technol. 51 (15), 8682-8691. doi:10.1021/acs.est.7b01133

Avellan, A., Yun, J., Morais, B. P., Clement, E. T., Rodrigues, S. M., and Lowry, G. V. (2021). Critical Review: Role of Inorganic Nanoparticle Properties on Their Foliar Uptake and in Planta Translocation. Environ. Sci. Technol. doi:10.1021/ acs.est.1c00178

Avellan, A., Yun, J., Zhang, Y., Spielman-Sun, E., Unrine, J. M., Thieme, J., et al. (2019). Nanoparticle Size and Coating Chemistry Control Foliar Uptake Pathways, Translocation, and Leaf-To-Rhizosphere Transport in Wheat. Acs Nano 13 (5), 5291-5305. doi:10.1021/acsnano.8b09781

Beckett, K. P., Freer-Smith, P. H., and Taylor, G. (2000b). Particulate Pollution Capture by Urban Trees: Effect of Species and Windspeed. Glob. Change Biol. 6, 995-1003. doi:10.1046/j.1365-2486.2000.00376.x

Beckett, K. P., Freer-Smith, P. H., and Taylor, G. (2000a). The Capture of Particulate Pollution by Trees at Five Contrasting Urban Sites. Arboricultural J. 24, 209-230. doi:10.1080/03071375.2000.9747273

Biasioli, M., Grčman, H., Kralj, T., Madrid, F., Díaz-Barrientos, E., and AjmoneMarsan, F. (2007). Potentially Toxic Elements Contamination in Urban Soils. J. Environ. Qual. 36, 70-79. doi:10.2134/jeq2006.0254

Conti, M. E., and Cecchetti, G. (2001). Biological Monitoring: Lichens as Bioindicators of Air Pollution Assessment - a Review. Environ. Pollut. 114 (3), 471-492. doi:10.1016/S0269-7491(00)00224-4

Douay, F., Pruvot, C., Waterlot, C., Fritsch, C., Fourrier, H., Loriette, A., et al. (2009). Contamination of Woody Habitat Soils Around a Former lead Smelter in the North of France. Sci. Total Environ. 407 (21), 5564-5577. doi:10.1016/ j.scitotenv.2009.06.015

Eichert, T., and Goldbach, H. E. (2008). Equivalent Pore Radii of Hydrophilic Foliar Uptake Routes in Stomatous and Astomatous Leaf Surfaces - Further Evidence for a Stomatal Pathway. Physiol. Plant 132 (4), 491-502. doi:10.1111/ j.1399-3054.2007.01023.x

Eichert, T., Kurtz, A., Steiner, U., and Goldbach, H. E. (2008). Size Exclusion Limits and Lateral Heterogeneity of the Stomatal Foliar Uptake Pathway for Aqueous Solutes and Water-Suspended Nanoparticles. Physiol. Plant. 134 (1), 151-160. doi:10.1111/j.1399-3054.2008.01135.x

Honour, S. L., Bell, J. N. B., Ashenden, T. W., Cape, J. N., and Power, S. A. (2009). Responses of Herbaceous Plants to Urban Air Pollution: Effects on Growth, Phenology and Leaf Surface Characteristics. Environ. Pollut. 157 (4), 1279-1286. doi:10.1016/j.envpol.2008.11.049

Hu, P., An, J., Faulkner, M. M., Wu, H., Li, Z., Tian, X., et al. (2020). Nanoparticle Charge and Size Control Foliar Delivery Efficiency to Plant Cells and Organelles. Acs Nano 14 (7), 7970-7986. doi:10.1021/ acsnano.9b09178

Hutchinson, T. C., and Whitby, L. M. (1974). Heavy-metal Pollution in the Sudbury Mining and Smelting Region of Canada, I. Soil and Vegetation Contamination by Nickel, Copper, and Other Metals. Envir. Conserv. 1 (2), 123-132. doi:10.1017/s0376892900004240

Kampschulte, M., Langheinirch, A., Sender, J., Litzlbauer, H., Althöhn, U., Schwab, J., et al. (2016). Nano-Computed Tomography: Technique and Applications. Fortschr Röntgenstr 188 (2), 146-154. doi:10.1055/s-0041106541

Kim, K., Seo, E., Chang, S.-K., Park, T. J., and Lee, S. J. (2016). Novel Water Filtration of saline Water in the Outermost Layer of Mangrove Roots. Sci. Rep. 6, 20426. doi:10.1038/srep20426

\section{SUPPLEMENTARY MATERIAL}

The Supplementary Material for this article can be found online at: https://www.frontiersin.org/articles/10.3389/fenvs.2021.702490/ full\#supplementary-material

Lee, S. J., Kim, H. N., Choi, W., Yoon, G. Y., and Seo, E. (2019). A Nature-Inspired Lubricant-Infused Surface for Sustainable Drag Reduction. Soft Matter 15 (42), 8459-8467. doi:10.1039/c9sm01576k

Little, P. E. (1995). Deposition of Exhaust lead and its Impact on Plants. Environmental and Medical Services Division. Oxford Shire, U.K: A.E.R.E., Didcot.

Lobersli, E. M., and Steinnes, E. (1988). Metal Uptake in Plants from a Birch Forest Area Near a Copper Smelter in Norway. Water Air Soil Pollut. 37 (1-2), 25-39.

Lough, T. J., and Lucas, W. J. (2006). Integrative Plant Biology: Role of Phloem Long-Distance Macromolecular Trafficking. Annu. Rev. Plant Biol. 57, 203-232. doi:10.1146/annurev.arplant.56.032604.144145

Luo, C., Liu, C., Wang, Y., Liu, X., Li, F., Zhang, G., et al. (2011). Heavy Metal Contamination in Soils and Vegetables Near an E-Waste Processing Site, south China. J. Hazard. Mater. 186, 481-490. doi:10.1016/j.jhazmat.2010.11.024

Lv, J., Christie, P., and Zhang, S. (2019). Uptake, Translocation, and Transformation of Metal-Based Nanoparticles in Plants: Recent Advances and Methodological Challenges. Environ. Sci. Nano 6 (1), 41-59. doi:10.1039/c8en00645h

Pollard, M., Beisson, F., Li, Y., and Ohlrogge, J. B. (2008). Building Lipid Barriers: Biosynthesis of Cutin and Suberin. Trends Plant Sci. 13 (5), 236-246. doi:10.1016/j.tplants.2008.03.003

Popp, C., Burghardt, M., Friedmann, A., and Riederer, M. (2005). Characterization of Hydrophilic and Lipophilic Pathways of Hedera helix L. Cuticular Membranes: Permeation of Water and Uncharged Organic Compounds. J. Exp. Bot. 56 (421), 2797-2806. doi:10.1093/jxb/eri272

Pourret, O., and Hursthouse, A. (2019). It's Time to Replace the Term "Heavy Metals" with "Potentially Toxic Elements" when Reporting Environmental Research. Int. J. Environ. Res. Public Health 16 (22), 4446. doi:10.3390/ ijerph16224446

Pourrut, B., Shahid, M., Dumat, C., Winterton, P., and Pinelli, E. (2011). Lead Uptake, Toxicity, and Detoxification in Plants. Rev. Environ. Contam. Toxicol. 213, 113-136. doi:10.1007/978-1-4419-9860-6_4

Salim, R., Al-Subu, M. M., and Atallah, A. (1993). Effects of Root and Foliar Treatments with Lead, Cadmium, and Copper on the Uptake Distribution and Growth of Radish Plants. Environ. Int. 19 (4), 393-404. doi:10.1016/01604120(93)90130-A

Schreck, E., Foucault, Y., Sarret, G., Sobanska, S., Cécillon, L., Castrec-Rouelle, M., et al. (2012). Metal and Metalloid Foliar Uptake by Various Plant Species Exposed to Atmospheric Industrial Fallout: Mechanisms Involved for lead. Sci. Total Environ. 427-428, 253-262. doi:10.1016/j.scitotenv.2012.03.051

Seo, E., Yoon, G. Y., Kim, H. N., Lim, J. H., Kim, S., Kim, B., et al. (2020). Morphological Features of Mucous Secretory Organ and Mucous Secretion of loachMisgurnus Anguillicaudatusskin for Friction Drag Reduction. J. Fish. Biol. 96 (1), 83-91. doi:10.1111/jfb.14186

Shahid, M., Dumat, C., Khalid, S., Schreck, E., Xiong, T., and Niazi, N. K. (2017). Foliar Heavy Metal Uptake, Toxicity and Detoxification in Plants: A Comparison of Foliar and Root Metal Uptake. J. Hazard. Mater. 325, 36-58. doi:10.1016/j.jhazmat.2016.11.063

Shahid, M., Xiong, T., Castrec-Rouelle, M., Leveque, T., and Dumat, C. (2013). Water Extraction Kinetics of Metals, Arsenic and Dissolved Organic Carbon from Industrial Contaminated poplar Leaves. J. Environ. Sci. 25 (12), 2451-2459. doi:10.1016/S1001-0742(12)60197-1

Sloof, J. E. (1993). Environmental Lichenology: Biomonitoring Trace-Element Air Pollution. Doctoral thesis. Delft: Technische Universiteit Delft.

Tomašević, M., Vukmirović, Z., Rajšić, S., Tasić, M., and Stevanović, B. (2005). Characterization of Trace Metal Particles Deposited on Some Deciduous Tree Leaves in an Urban Area. Chemosphere 61 (6), 753-760. doi:10.1016/ j.chemosphere.2005.03.077 
Uzu, G., Sobanska, S., Sarret, G., Muñoz, M., and Dumat, C. (2010). Foliar Lead Uptake by Lettuce Exposed to Atmospheric Fallouts. Environ. Sci. Technol. 44 (3), 1036-1042. doi:10.1021/es902190u

Wang, W.-N., Tarafdar, J. C., and Biswas, P. (2013). Nanoparticle Synthesis and Delivery by an Aerosol Route for Watermelon Plant Foliar Uptake. J. Nanopart Res. 15 (1), 1417. doi:10.1007/s11051013-1417-8

Ward, N. I. (1990). Lead Contamination of the London Orbital (M25) Motorway (Since its Opening in 1986). Sci. Total Environ. 93, 277-283. doi:10.1016/00489697(90)90117-D

Wolterbeek, B. (2002). Biomonitoring of Trace Element Air Pollution: Principles, Possibilities and Perspectives. Environ. Pollut. 120 (1), 11-21. doi:10.1016/ s0269-7491(02)00124-0

Xiong, T.-T., Leveque, T., Austruy, A., Goix, S., Schreck, E., Dappe, V., et al. (2014). Foliar Uptake and Metal(loid) Bioaccessibility in Vegetables Exposed to Particulate Matter. Environ. Geochem. Health 36 (5), 897-909. doi:10.1007/ s10653-014-9607-6
Conflict of Interest: The authors declare that the research was conducted in the absence of any commercial or financial relationships that could be construed as a potential conflict of interest.

Publisher's Note: All claims expressed in this article are solely those of the authors and do not necessarily represent those of their affiliated organizations, or those of the publisher, the editors and the reviewers. Any product that may be evaluated in this article, or claim that may be made by its manufacturer, is not guaranteed or endorsed by the publisher.

Copyright (c) 2021 Seo, Kim, Park, Lim, Ha, Lim, Lim, Park, Kim and Lee. This is an open-access article distributed under the terms of the Creative Commons Attribution License (CC BY). The use, distribution or reproduction in other forums is permitted, provided the original author(s) and the copyright owner(s) are credited and that the original publication in this journal is cited, in accordance with accepted academic practice. No use, distribution or reproduction is permitted which does not comply with these terms. 